\title{
Exponential Stability for Impulsive Stochastic Nonlinear Network Systems with Time Delay
}

\author{
Lanping Chen, ${ }^{1,2}$ Zhengzhi Han, ${ }^{1}$ and Zhenghua $\mathrm{Ma}^{2}$ \\ ${ }^{1}$ School of Electronic, Information and Electrical Engineering, Shanghai Jiaotong University, Shanghai 200240, China \\ ${ }^{2}$ College of Information and Engineering Science, Changzhou University, Jiangsu 213164, China \\ Correspondence should be addressed to Lanping Chen; lanping.chen@gmail.com
}

Received 4 December 2013; Revised 29 January 2014; Accepted 6 February 2014; Published 17 March 2014

Academic Editor: Laurence T. Yang

Copyright (c) 2014 Lanping Chen et al. This is an open access article distributed under the Creative Commons Attribution License, which permits unrestricted use, distribution, and reproduction in any medium, provided the original work is properly cited.

\begin{abstract}
We study the exponential stability of the complex dynamical network described by differentially nonlinear equations which couple with time delay and stochastic impulses. Some sufficient conditions are established to ensure $p$ th moment exponential stable for the stochastic impulsive systems (SIS) with time delay. An example with its numerical simulation is presented to illustrate the validation of main results.
\end{abstract}

\section{Introduction}

As the extension and expansion of Internet network, the Internet of things is the complex networks which are made up of interconnected nodes and used to describe various systems of real world. In many systems such as signal processing systems, computer networks, automatic control systems, flying object motions, and telecommunications, impulsive effects are common phenomena due to instantaneous perturbations at certain moments. Therefore, the study of the dynamical networks with impulsive effects is important for understanding the dynamical behaviors of the most real-world complex networks. The impulsive dynamic systems have been studied extensively (see [1-4] and references therein). In addition to impulsive effects, stochastic effects likewise exist in real systems. In recent years stochastic impulsive dynamic system is an emerging field drawing attention from various disciplines of sciences and engineering.

Many real-world problems in science and engineering can be modeled by nonlinear stochastic impulsive dynamic systems (see $[5,6]$ and references therein). The stability analysis is much more complicated because of the existence of simultaneous impulsive effects and stochastic effects. So far, there are several results on impulsive stochastic systems, which we can find in [7-10]. However, to the best of the authors' knowledge, little study on impulsive stabilization of stochastic delay systems has been done so far. Motivated by the above consideration, in this paper we analysis this system and obtain sufficient conditions to ensure the $p$ th moment asymptotic stability of stochastic impulsive systems with arbitrarily infinite delays. It is shown that an unstable stochastic delay system can be successfully stabilized by impulses and the results can be easily applied to stochastic systems with arbitrarily time delays.

\section{Preliminaries}

Let $R^{n}$ denote the $n$-dimensional real space and let $\tau>0$ be a positive real number. Let $\mathrm{PC}\left([-\tau, 0] ; R^{n}\right)$ denotes the family of piecewise continuous functions from $[-\tau, 0]$ to $R^{n}$. $\mathrm{PC}\left([-\tau, 0] ; R^{n}\right)=\varphi:[-\tau, 0] \rightarrow R^{n} \mid \varphi\left(t^{+}\right)=\varphi(t), \varphi(t)$ exists, and $\varphi\left(t^{-}\right)=\varphi(t)$ for $t \in(-\tau, 0]$, with the norm $\|\varphi\|=$ $\sup _{-\tau \leq \theta \leq 0}|\varphi(\theta)|$, where $\varphi\left(t^{+}\right)$and $\varphi\left(t^{-}\right)$denote the right-hand and left-hand limit of function $\varphi(t)$ at $t$, respectively.

Consider the impulsive stochastic differential equation as follows:

$d x(t)=f\left(t, x(t), x_{t}\right) d t+g\left(t, x_{t}\right) d w(t), \quad t \geq 0, t \neq t_{k}$, 


$$
\begin{gathered}
\Delta x\left(t_{k}\right)=x\left(t_{k}^{+}\right)-x\left(t_{k}\right)=I_{k}\left(x\left(t_{k}\right)\right), \\
t=t_{k}, \quad k=1,2, \ldots, m, \\
x\left(t_{0}\right)=\xi, \quad t=[-\tau, 0],
\end{gathered}
$$

where the initial value $\xi \in \mathrm{PC}\left([-\tau, 0] ; R^{n}\right), x(t)=$ $\left[x_{1}(t), x_{2}(t), \ldots, x_{n}(t)\right]^{T}, \quad x_{t}$ is regarded as a PC-valued stochastic process, $\left.x_{t}=\{x(t+\theta):-\tau \leq \theta \leq 0)\right\}, f: R^{+} \times R^{n} \times$ $\mathrm{PC}\left([-\tau, 0] ; R^{n}\right) \rightarrow R^{n}, g: R^{+} \times \mathrm{PC}\left([-\tau, 0] ; R^{n}\right) \rightarrow R^{n \times m}$, and $w(t)$ is an $m$-dimensional standard Brownian motion defined on the complete probability space.

Definition 1. Let $C^{2,1}\left(R^{n} \times\left[t_{0}-\tau, \infty\right) ; R^{+}\right)$denote the family of all nonnegative functions $V(x, t)$ on $R^{n} \times\left[t_{0}-\tau, \infty\right)$ that are continuously twice differentiable in $x$ and once in $t$. For a $V \in C^{2,1}\left(R^{n} \times\left[t_{0}-\tau, \infty\right) ; R^{+}\right)$, one can define the Kolmogorov operator $\mathscr{L} \mathrm{V}$ as follows:

$$
\begin{aligned}
\mathscr{L} V(x, t)= & V_{t}(x, t)+V_{x}(x, t) f(x, t) \\
& +\frac{1}{2} \operatorname{Tr}\left\{g^{T}(x, t) V_{x x} g(x, t)\right\},
\end{aligned}
$$

where $V_{t}=\partial V(x, t) / \partial t, V_{x}=\left(\partial V(x, t) / \partial x_{1}, \ldots, \partial V(x, t) / \partial x_{n}\right)$, and $V_{x x}=\partial^{2} V(x, t) / \partial x^{2}$.

Definition 2. The trivial solution of SIS (1) is said to be the $p$ th moment exponential stable if there exist positive constants $\alpha>0$ and $K \geq 1$ such that

$$
E\|x(t)\|^{p} \leq K e^{-\alpha\left(t-t_{0}\right)}\left\|x_{0}\right\|^{p}, \quad t>t_{0}, t \in R^{+} .
$$

The following lemmas can be found in [11].

Lemma 3. Let $x, y \geq 0, a, b>1$, then

$$
x y \leq \frac{x^{a}}{a}+\frac{y^{b}}{b}, \quad \frac{1}{a}+\frac{1}{b}=1 .
$$

Lemma 4. Let $x, y \geq 0, p \geq j \geq 0$, then

$$
x^{p-j} y^{j} \leq \frac{(p-j) x^{p}+j y^{p}}{p} .
$$

\section{Main Results}

In this section, we shall focus on sufficient conditions to achieve exponential stability of the SIS by employing Razumikhin techniques and Lyapunov functions. Moreover, we will design the impulsive control for the stabilization of unstable stochastic systems by using the obtained results.

Theorem 5. If there exist positive constants $p, c_{1}, c_{2}, \lambda, d_{k}>1$, and suppose there exists a function $V$ such that

(i) $c_{1}|x|^{p} \leq V(x, t) \leq c_{2}|x|^{p}$;

(ii) $E V\left(x, t_{k}^{+}\right) \leq d_{k} E V\left(x, t_{k}\right)$; (iii) $\operatorname{ELV}(\varphi, t) \leq c E V(\varphi, t)$, for all $t \in\left(t_{k-1}, t_{k}\right]$;

(iv) $\ln d_{k} \leq \lambda\left(t_{k}-t_{k-1}\right), k=1,2, \ldots$.

Then the corresponding system (1) is the pth moment exponential stable.

Proof. For any $t \in\left[t_{1}, t_{2}\right]$, we can get from the conditions (ii) and (iii)

$$
\begin{aligned}
E V(t)= & E V\left(t_{1}^{+}\right)+\int_{t_{1}}^{t} c E V(x(s), s) d s \\
\leq & d_{1}\left[E V(0)+\int_{0}^{t_{1}} c E V(x(s), s) d s\right] \\
& +\int_{t_{1}}^{t} c E V(x(s), s) d s=d_{1} E V(0) \\
& +d_{1} \int_{0}^{t_{1}} c E V(x(s), s) d s+\int_{t_{1}}^{t} c E V(x(s), s) d s
\end{aligned}
$$

In general for $t \in\left[t_{k-1}, t_{k}\right]$, one can find that

$$
E V(t) \leq \prod_{0 \leq t_{k} \leq t} d_{k} E V(0)+\int_{0}^{t} \prod_{s \leq t_{k} \leq t} d_{k} c E V(x(s), s) d s .
$$

From condition (iv), we get

$$
\begin{aligned}
\prod_{s \leq t_{k} \leq t} d_{k} & \leq e^{\lambda\left(t_{2}-t_{1}\right)} \cdot e^{\lambda\left(t_{3}-t_{2}\right)} \cdots e^{\lambda\left(t_{k}-t_{k-1}\right)} \\
& =e^{\lambda\left(t_{k}-t_{1}\right)}=e^{\lambda(t-s)} \cdot e^{\lambda\left(t_{k}-t\right)} \cdot e^{\lambda\left(s-t_{1}\right)} .
\end{aligned}
$$

For $t \in\left[t_{k-1}, t_{k}\right], t_{1}, t_{2}, \ldots, t_{k}$ be impulsive points in $[s, t), t>$ $s, \lambda<0$, then we obtain

$$
\prod_{s \leq t_{k} \leq t} d_{k} \leq e^{\lambda(t-s)} \cdot e^{\lambda\left(t_{k}-t\right)} \leq e^{\lambda(t-s)} \cdot e^{\lambda\left(t_{k}-t_{k-1}\right)} \leq \gamma e^{\lambda(t-s)} .
$$

By (7) and (8), then we can get

$$
\begin{aligned}
E V(t) & \leq \gamma E V(0) e^{\lambda t}+\int_{0}^{t} \gamma e^{\lambda(t-s)} c E V(x(s), s) d s \\
& \leq \gamma \sup _{-\tau \leq \theta \leq 0} E V(\sigma) e^{\lambda t} .
\end{aligned}
$$

It follows from condition (i), that

$$
c_{1} E|x(t)|^{p} \leq E V(t) \leq \gamma \sup _{-\tau \leq \theta \leq 0} E V(\sigma) e^{\lambda t} \leq \gamma c_{2} E\|\xi\|^{p} e^{\lambda t},
$$

which implies

$$
E|x(t)|^{p} \leq \frac{\gamma c_{2}}{c_{1}} E\|\xi\|^{p} e^{\lambda t} .
$$

System (1) is the pth moment exponentially stable. The proof is complete. 
Theorem 6. Assume that

(i) $E V_{t} \leq \eta E V(x(t)), E V_{x} \leq \overline{\eta_{1}} E V(x(t))^{(p-1) / p}, E V_{x x} \leq$ $\overline{\eta_{2}} E V(x(t))^{(p-2) / p}$

(ii) $E\left|f\left(x_{t}\right)\right|^{p} \quad \leq \quad \overline{\eta_{3}} \sup _{-\tau \leq \theta \leq 0} E V\left(x_{t}\right)$, $E\left(\operatorname{Tr}\left\{g\left(x_{t}\right)^{T} g\left(x_{t}\right)\right\}\right)^{p / 2} \leq \overline{\eta_{4}} \sup _{-\tau \leq \theta \leq 0} E V(x(t-\tau)) ;$

(iii) $E V(x(t+\theta)) \leq q E V(x(t)), \theta \in(-\tau, 0]$;

and conditions of Theorem 5 hold simultaneously, then the system is pth exponential stable, where $E$ denotes the expectation.

Proof. Take

$$
\mathscr{L} V=V_{t}+V_{x} f\left(x_{t}\right)+\frac{1}{2} \operatorname{Tr}\left\{g^{T}\left(x_{t}\right) V_{x x} g\left(x_{t}\right)\right\}
$$

then take the mathematical expectation of both sides of the Formula (12), we obtain

$$
E \mathscr{L} V=E V_{t}+E\left(V_{x} f\right)+\frac{1}{2} E\left[\operatorname{Tr}\left\{g^{T} V_{x x} g\right\}\right] .
$$

Using Lemma 3, from (i) and (ii), we obtain

$$
\begin{aligned}
& E\left(V_{x} f\right.\left.\left(x, x_{t}\right)\right) \\
& \leq E V_{x} \cdot E f\left(x, x_{t}\right) \leq \overline{\eta_{1}} E(V)^{(p-1) / p} \cdot E\left|f\left(x, x_{t}\right)\right| \\
& \leq \frac{\overline{\eta_{1}}(p-1)}{p} E V+\frac{1}{p} \overline{\eta_{3}} \cdot E\left|f\left(x, x_{t}\right)\right|^{p} \leq \frac{\overline{\eta_{1}}(p-1)}{p} E V \\
&+\frac{1}{p} \overline{\eta_{3}} \cdot q \cdot \sup _{-\tau \leq \theta \leq 0} E V(x(t)), \\
& E\left[\operatorname{Tr}\left\{g^{T}\left(x_{t}\right) V_{x x} g\left(x_{t}\right)\right\}\right] \\
& \leq E V_{x x} \cdot E\left[\operatorname{Tr}\left\{g^{T}\left(x_{t}\right) g\left(x_{t}\right)\right\}\right] \leq \overline{\eta_{2}} E(V(x))^{(p-2) / p} \\
& \cdot E\left(\operatorname{Tr}\left\{g^{T}\left(x_{t}\right) g\left(x_{t}\right)\right\}\right) \leq \frac{\overline{\eta_{2}}(p-2)}{p} E V(x) \\
&+\frac{2}{p} E\left[\operatorname{Tr}\left\{g^{T} g\right\}\right]^{p / 2} \leq \frac{\overline{\eta_{2}}(p-2)}{p} E V(x) \\
&+\frac{2 \overline{\eta_{4}}}{p} \cdot \sup _{-\tau \leq \theta \leq 0} E V(x(t-\tau)) \leq \frac{\overline{\eta_{2}}(p-2)}{p} E V(x) \\
&+\frac{2 \overline{\eta_{4}}}{p} \cdot q \cdot \sup _{-\tau \leq \theta \leq 0} E V(x(t)) .
\end{aligned}
$$

It follows that

$$
\begin{aligned}
E \mathscr{L} V \leq & \overline{\eta_{1}} E V(x(t))+\frac{\overline{\eta_{1}}(p-1)}{p} E V(x(t)) \\
& +\frac{\overline{\eta_{2}}(p-2)}{p} E V(x)+\frac{1}{p} \overline{\eta_{3}} \cdot q \cdot \sup _{-\tau \leq \theta \leq 0} E V(x(t)) \\
& +\frac{2 \overline{\eta_{4}}}{p} \cdot q \cdot \sup _{-\tau \leq \theta \leq 0} E V(x(t)) .
\end{aligned}
$$

Consequently, by the above statement, the conditions of Theorem 5 are all satisfied. Then, the conclusion follows from Theorem 5 and the proof is complete.

Remark 7. From the above consequence, we know that the unstable stochastic system $d x(t)=f\left(t, x, x_{t}\right) d t+g\left(t, x_{t}\right)$ $d w(t)$ can be exponentially stabilized by the impulsive control $u_{k}(x)=I_{k}(x), t_{k}, k \in N$. Moreover, the steps of the impulsive control design satisfy the conditions of Theorem 5 .

Remark 8. Consider a special case of system (1) shown as follows:

$$
\begin{gathered}
d x(t)=\left[A x+f_{1}(t, x(t-\tau))\right] d t+g(t, x(t-\tau)) d w(t), \\
t \geq 0, \quad t \neq t_{k}, \\
\Delta x\left(t_{k}\right)=x\left(t_{k}^{+}\right)-x\left(t_{k}\right)=I_{k}\left(x\left(t_{k}\right)\right), \\
t=t_{k}, \quad k=1,2, \ldots, m, \\
x\left(t_{0}\right)=\xi, \quad t=[-\tau, 0],
\end{gathered}
$$

there exist nonnegative function $\alpha_{i}(t), \beta_{i}(t)$ such that

(i) $\left|f_{1}(t, x(t-\tau))\right| \leq \sum_{i=1}^{m} \alpha_{i}(t)\left|x\left(t-\tau_{i}\right)\right|$,

(ii) $\|g(t, x(t-\tau))\|_{F}^{2} \leq \sum_{i=1}^{m} \beta_{i}(t)\left|x\left(t-\tau_{i}\right)\right|^{2}$,

where $\tau=\max _{1 \leq i \leq m} \tau_{i}, \lambda_{\max }(\cdot)$ denotes the largest eigenvalue of a symmetric matrix. Then we derive the following theorem.

Theorem 9. Assume that there exist positive constants $\kappa_{1}, \kappa_{2}$, $\kappa_{3}$ such that

$$
\begin{gathered}
\kappa_{1}+(p-1) \kappa_{2}+\frac{(p-2) \kappa_{3}}{2}>0 \\
\kappa_{2}+\kappa_{3}>0
\end{gathered}
$$

hold, where $\kappa_{1}=\lambda_{\max }(A), \kappa_{2}=\Theta_{i=1}^{m} \alpha_{i}(t), \kappa_{3}=(p-1)$ $\Theta_{i=1}^{m} \beta_{i}(t)$, and if the conditions of Theorems 5 and 6 are satisfied, then the trivial solution of system (17) is p-moment exponentially stable.

Proof. Let $P=Q^{T} Q$, and $V(t, x)=\left(x^{T} P x\right)^{p / 2}=|Q x|^{p}$. Then by Itô formula, we have

$$
\begin{aligned}
\mathscr{L} V= & V_{t}+V_{x}\left[A x+f_{1}(x(t-\tau))\right]+\frac{1}{2} \operatorname{Tr}\left\{g^{T} V_{x x} g\right\} \\
= & p|Q x|^{p-1}\left[Q A x+Q f_{1}(t, x(t-\tau))\right]+\frac{p(p-1)}{2} \\
& \times|Q x|^{p-2} \operatorname{Tr}\left\{g^{T}(t, x(t-\tau)) \operatorname{Pg}(t, x(t-\tau))\right\} .
\end{aligned}
$$

By condition (ii), we have

$$
\begin{aligned}
\operatorname{Tr}\left\{g^{T}(t, x(t-\tau)) P g(t, x(t-\tau))\right\} \\
\quad=\|Q g(t, x(t-\tau))\|_{F}^{2} \leq \Sigma_{i=1}^{m} \beta_{i}(t)\left|Q x\left(t-\tau_{i}\right)\right|^{2} .
\end{aligned}
$$


Substituting (20) into (19), and using conditions, we obtain

$$
\begin{aligned}
\mathscr{L} V \leq & p|Q x|^{p-1}\left[\lambda_{\max }(A)|Q x|+\sum_{i=1}^{m} \alpha_{i}(t)\left|Q x\left(t-\tau_{i}\right)\right|\right] \\
& +\frac{p(p-1)}{2}|Q x|^{p-2} \sum_{i=1}^{m} \beta_{i}(t)\left|Q x\left(t-\tau_{i}\right)\right|^{2} .
\end{aligned}
$$

Using Lemma 4, we get

$$
\begin{aligned}
\mathscr{L} V \leq|Q x|^{p}\left[\lambda_{\max }(A)+(p-1) \sum_{i=1}^{m} \alpha_{i}(t)\right. \\
\left.+\frac{(p-1)(p-2)}{2} \sum_{i=1}^{m} \beta_{i}(t)\right] \\
+\left[\sum_{i=1}^{m} \alpha_{i}(t)+(p-1) \sum_{i=1}^{m} \beta_{i}(t)\right]\left|Q x\left(t-\tau_{i}\right)\right|^{p} \\
=\left[\kappa_{1}+(p-1) \kappa_{2}+\frac{(p-2) \kappa_{3}}{2}\right] V(t, x) \\
+\left[\kappa_{2}+\kappa_{3}\right] V\left(t, x\left(t-\tau_{i}\right)\right) .
\end{aligned}
$$

Summing up the above statements, we can see that all the conditions of Theorem 5 and condition (iii) of Theorem 6 are satisfied. Then the conclusion follows from Theorem 6 immediately and the proof is completed.

\section{Example}

In this section, we present an example to demonstrate our theoretical results. Considering a nonlinear stochastic impulsive system as follows:

$$
\begin{gathered}
d x(t)=f(x(t)) d t+g(x(t), x(t-\tau)) d w(t), \\
t \geq 0, \quad t \neq t_{k}, \\
\Delta x\left(t_{k}\right)=I_{k}\left(x\left(t_{k}\right)\right), \quad t=t_{k}, \quad k \in N,
\end{gathered}
$$

where $f(x)=x(t), g\left(x, x_{t}\right) \leq(1 / 4)\left(x^{2}+x_{t}^{2}\right), I_{k}=-0.4, \tau=2$.

Step 1. Calculate the parameters.

Without loss of generality, we choose $c_{1}=c_{2}=1, p=2$, $d_{k}=0.37$ such that they satisfy the conditions of (i) and (ii) of Theorem 5 .

Step 2. Choose $V(x, t)=x^{2}$, then it is easy to calculate from the Itô formula that

$$
\begin{aligned}
E \mathscr{L} V(x(t), x(t-\tau)) & =2 E|x(t)|^{2}+E\left|g\left(x, x_{t}\right)\right|^{2} \\
& \leq \frac{9}{4} E V(x(t))+\frac{1}{4} E V(x(t-\tau))
\end{aligned}
$$

which satisfies condition (iii) of Theorem 5

$$
E \mathscr{L} V(x(t), x(t-\tau)) \leq c E V(x(t)),
$$

where take $q=5, \lambda=0.5, t_{k+1}-t_{k}=0.2$.

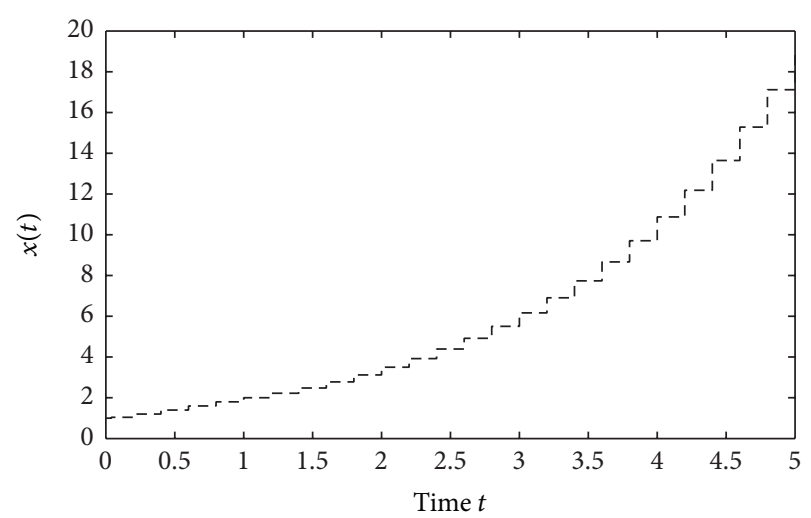

FIGURE 1: Instability of the stochastic delay system (23) without impulsive effect.

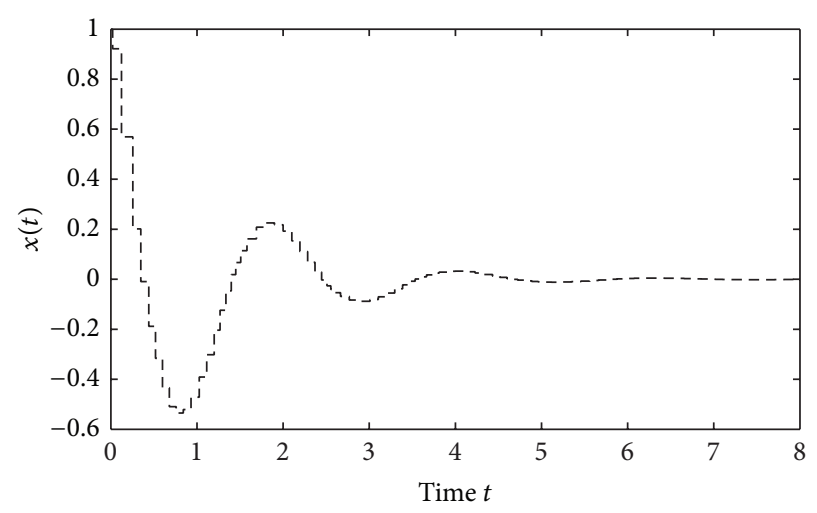

FIgURE 2: Impulsive Stabilization of the stochastic delay system (23).

Step 3. By calculation, we obtain $c=3.5$, then

$$
\ln d_{k}=-1.02<-(c+\lambda)\left(t_{k+1}-t_{k}\right)=-0.8 \text {. }
$$

It satisfies condition (iv) of Theorem 5 which means that the system (23) is exponentially stable. Figure 1 gives the trajectory of the state of (23). It is obvious that the system is not stable without impulsive effect. Figure 1 shows that the solution of the stochastic delayed system (23) is unstable. Figure 2 shows the stability of the delay system with the impulsive controller.

\section{Conclusion}

In this paper, we have investigated the $p$-moment stability and applied the technique of Razumikhin techniques and Lyapunov functions to impulsive stochastic systems. Some sufficient conditions about the stability of impulsive stochastic systems in terms of two measures are derived. As a beneficial supplement in the study of impulsive stochastic systems with time delay, the concluded criteria are not only effective but also convenient in practical applications of specific systems in engineering and physics, etc. We also provided an illustrative example to show the effectiveness of our results. 


\section{Conflict of Interests}

The authors declare that there is no conflict of interests regarding the publication of this paper.

\section{Acknowledgments}

The authors are grateful for the support of the National Natural Science Foundation of China (Grant no. 61074003). This work is supported by the National Natural Science Foundation of China (no. 61074003).

\section{References}

[1] S. Zhang, J. Sun, and Y. Zhang, "Stability of impulsive stochastic differential equations in terms of two measures via perturbing Lyapunov functions," Applied Mathematics and Computation, vol. 218, no. 9, pp. 5181-5186, 2012.

[2] Q. Wang and X. Liu, "Impulsive stabilization of delay differential systems via the Lyapunov-Razumikhin method," Applied Mathematics Letters, vol. 20, no. 8, pp. 839-845, 2007.

[3] X. Song and A. Li, "Stability and boundedness criteria of nonlinear impulsive systems employing perturbing Lyapunov functions," Applied Mathematics and Computation, vol. 217, no. 24, pp. 10166-10174, 2011.

[4] Y. Liu and S. Zhao, "A new approach to practical stability of impulsive functional differential equations in terms of two measures," Journal of Computational and Applied Mathematics, vol. 223, no. 1, pp. 449-458, 2009.

[5] K. Liu, Stability of Infinite Dimensional Stochastic Differential Equations with Applications, vol. 135, Chapman \& Hall/CRC, London, UK, 2006.

[6] L. Wan and J. Duan, "Exponential stability of non-autonomous stochastic partial differential equations with finite memory," Statistics \& Probability Letters, vol. 78, no. 5, pp. 490-498, 2008.

[7] P. Cheng, F.-Q. Deng, and X.-S. Da, "Razumikhin-type theorems for asymptotic stability of impulsive stochastic functional differential systems," Journal of Systems Science and Systems Engineering, vol. 19, no. 1, pp. 72-84, 2010.

[8] S. Peng and Y. Zhang, "Razumikhin-type theorems on pth moment exponential stability of impulsive stochastic delay differential equations," IEEE Transactions on Automatic Control, vol. 55, no. 8, pp. 1917-1922, 2010.

[9] B. Liu, "Stability of solutions for stochastic impulsive systems via comparison approach," IEEE Transactions on Automatic Control, vol. 53, no. 9, pp. 2128-2133, 2008.

[10] X. Mao, G. G. Yin, and C. Yuan, "Stabilization and destabilization of hybrid systems of stochastic differential equations," Automatica, vol. 43, no. 2, pp. 264-273, 2007.

[11] Q. Song and Z. Wang, "Stability analysis of impulsive stochastic Cohen-Grossberg neural networks with mixed time delays," Physica A, vol. 387, no. 13, pp. 3314-3326, 2008. 


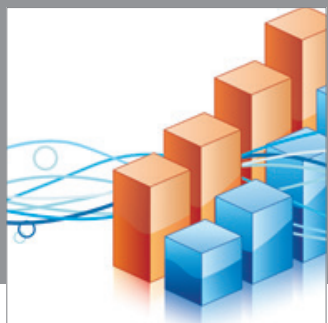

Advances in

Operations Research

mansans

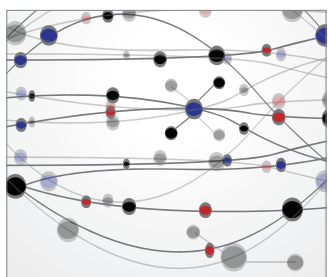

The Scientific World Journal
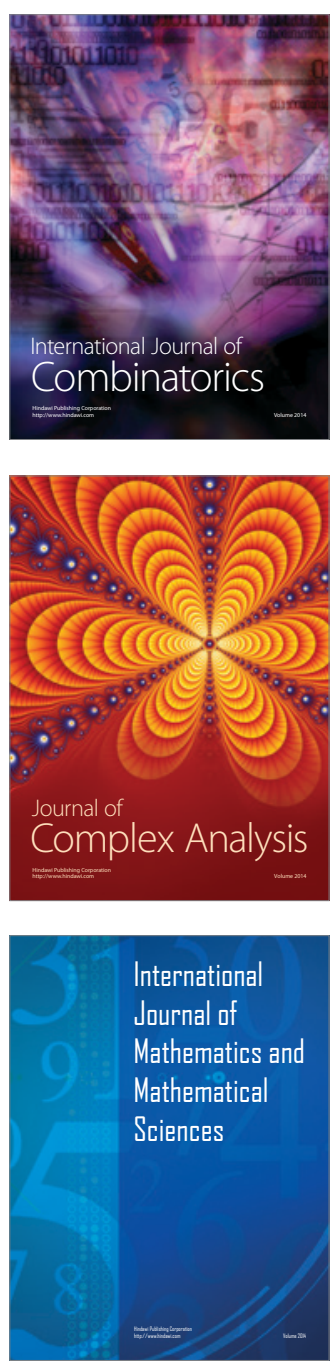
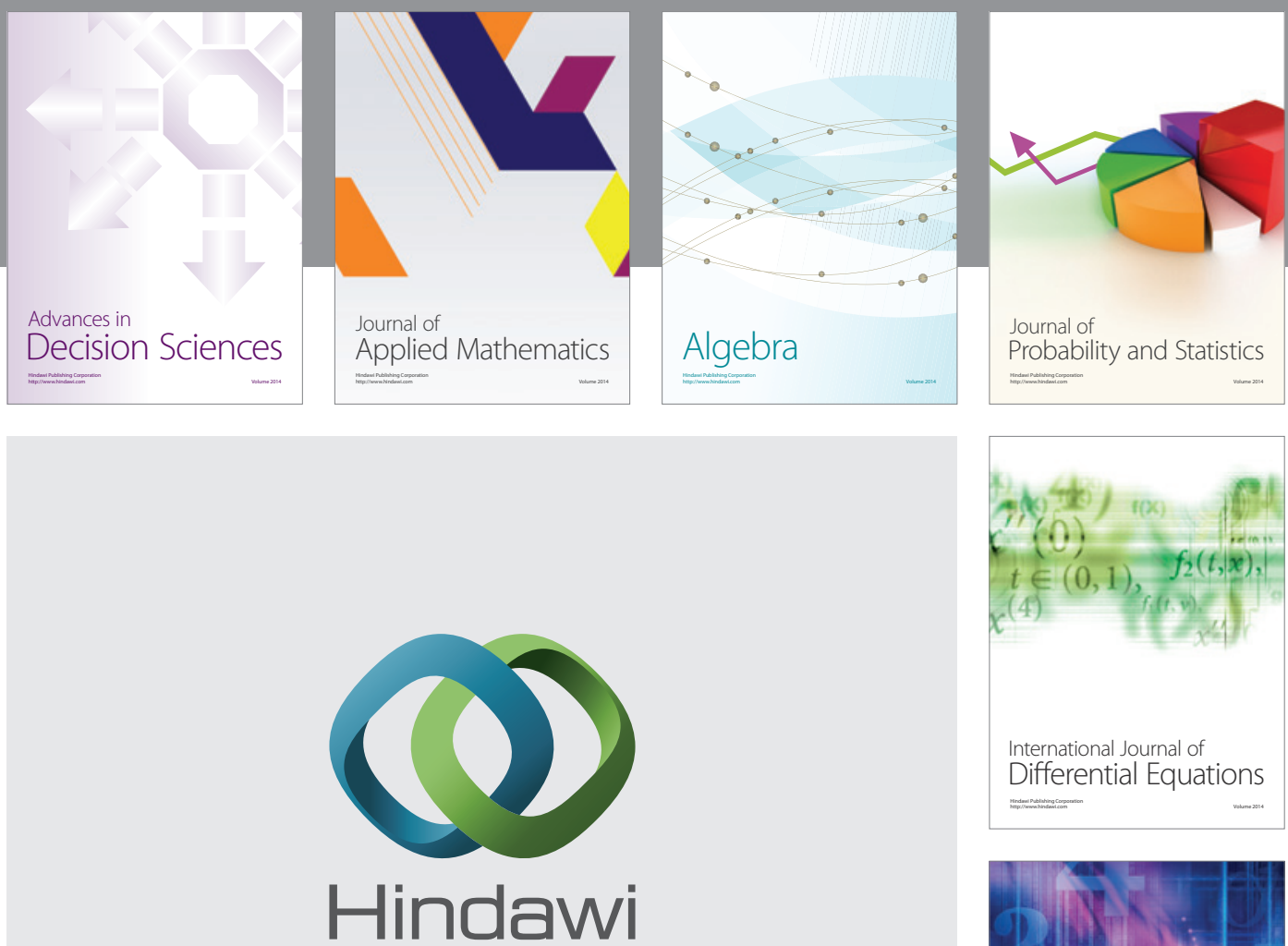

Submit your manuscripts at http://www.hindawi.com
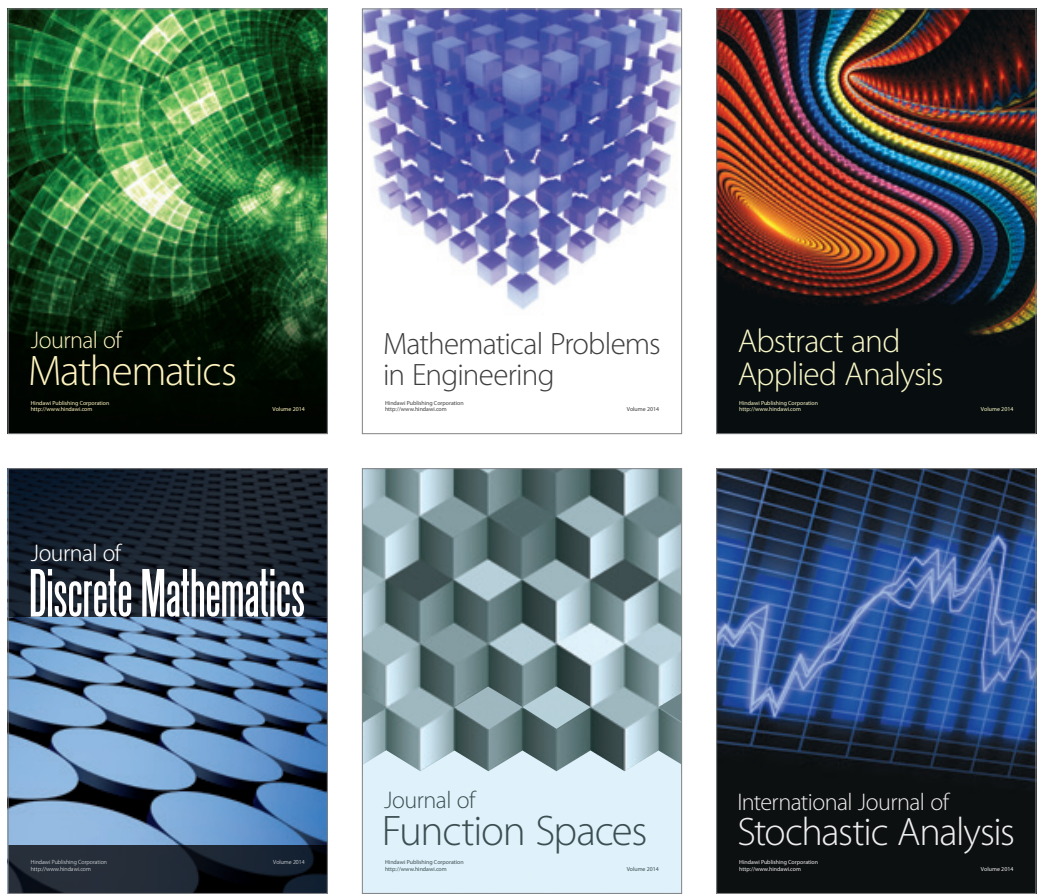

Journal of

Function Spaces

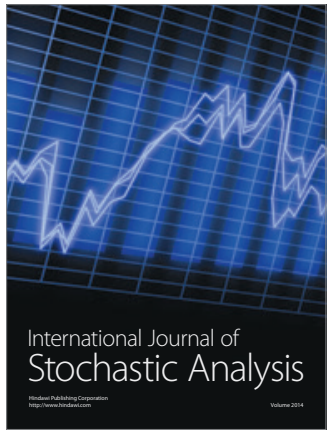

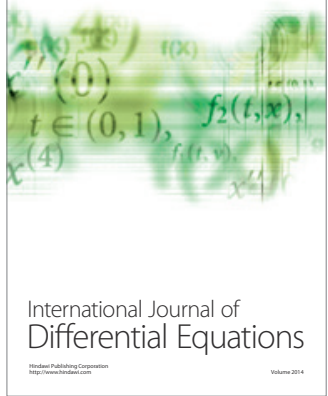
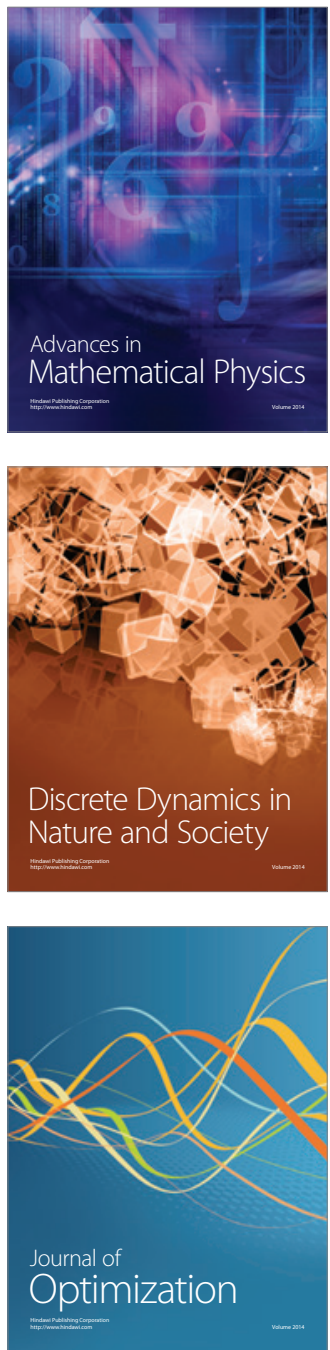